\title{
ON NON-MIDPOINT LOCALLY UNIFORMLY ROTUND NORMABILITY IN BANACH SPACES
}

\author{
A. K. MIRMOSTAFAEE
}

Received 21 May 2002

\begin{abstract}
We will show that if $X$ is a tree-complete subspace of $\ell_{\infty}$, which contains $c_{0}$, then it does not admit any weakly midpoint locally uniformly convex renorming. It follows that such a space has no equivalent Kadec renorming.
\end{abstract}

2000 Mathematics Subject Classification: 46B20.

1. Introduction. It is known that $\ell_{\infty}$ has an equivalent strictly convex renorming [2]; however, by a result due to Lindenstrauss, it cannot be equivalently renormed in locally uniformly convex manner [10]. In this note, we will show that every tree-complete subspace of $\ell_{\infty}$, which contains $c_{0}$, does not admit any equivalent weakly midpoint locally uniformly convex norm. This can be considered as an extension of $[1,8]$. Since every strictly convexifiable Banach space with Kadec property admits an equivalent midpoint locally uniformly convex renorming [9], it follows that every subspace of $\ell_{\infty}$ with the tree-completeness property has no equivalent Kadec renorming. The existence of such a (nontrivial) subspace, which does not contain any copy of $\ell_{\infty}$, has already been proved by Haydon and Zizler (see [5, 7]).

2. Results. We recall that a norm $\|\cdot\|$ on a Banach space $X$ is said to be midpoint locally uniformly rotund (MLUR) if, whenever $\left\{x_{n}\right\},\left\{y_{n}\right\}$, and $x$ are in $X$ with $\left\|x_{n}\right\| \rightarrow$ $\|x\|,\left\|y_{n}\right\| \rightarrow\|x\|$, and $\left\|\left(x_{n}+y_{n}\right) / 2-x\right\| \rightarrow 0$, we necessarily have $\left\|x_{n}-y_{n}\right\| \rightarrow 0$. If at the end of the last sentence, we replace norm with weak, the definition of weakly midpoint locally uniformly rotund (wMLUR) will be obtained [3]. Let $T$ be the set of all finite (possible empty) strings of 0's and 1's. The empty string ( ) is the unique string of length 0 ; the length $|t|$ of a string $t$ is $n$ if $t \in\{0,1\}^{n}$. The tree order is defined by $s \prec t$ if $|s|<|t|$ and $t(m)=s(m)$ for $m \leq|s|$. Each $t \in T$ has exactly two immediate successors, that is, $t 0$ and $t 1$.

A lattice $L$ is said to be tree-complete if, whenever $\left\{f_{t}\right\}_{t \in T}$ is a bounded disjoint family in $L$, there exists $b \in\{0,1\}^{N}$, such that $\sum_{n \in N} f_{b \mid n}$ is in $L$.

Haydon and Zizler [7] constructed a closed linear subspace of $\ell_{\infty}$ (which is a treecomplete sublattice of $\ell_{\infty}$ ) such that it contains $c_{0}$ but does not contain any subspace isomorphic to $\ell_{\infty}$. Notice that in this space $X$ every infinite subset $M$ of $N$ has an infinite subset $M_{0} \subset M$ such that $\mathbf{1}_{M_{0}} \in X$ [7].

THEOREM 2.1. Let $X$ be a tree-complete sublattice of $\ell_{\infty}$. If $X$ contains $c_{0}$, then $X$ does not admit any equivalent wMLUR renorming. 
Proof. Let $\||\cdot|\|$ be an equivalent norm on $X$. We will show that this norm is not wMLUR. Let

$$
\begin{gathered}
A_{()}=\left\{f \in X:\|f\|_{\infty}=1, N \backslash \operatorname{supp}(f) \text { is infinite }\right\}, \\
M_{()}=\sup \left\{\|\| f \|: \mid f \in A_{()}\right\}, \quad m_{()}=\inf \left\{\||| f\| \mid \|: f \in A_{())}\right\} .
\end{gathered}
$$

Choose an element $f_{()}$of $X$ such that $\left\|\mid f_{()}\right\| \|>\left(3 M_{()}+m_{()}\right) / 4$. Then select two disjoint infinite subsets $N_{0}^{\prime}$ and $N_{1}^{\prime}$ of $N \backslash \operatorname{supp}\left(f_{()}\right)$with $\mathbf{1}_{N_{i}^{\prime}} \in X$ for some $k_{i} \in N_{i}^{\prime}$, define $N_{i}=N_{i}^{\prime} \backslash\left\{k_{i}\right\}$, and let

$$
A_{i}=\left\{f \in A_{()}: f(n)=f_{(}(n) \text { for each } n \notin N_{i}\right\} \quad(i=0,1) .
$$

Suppose that for some $t \in T$, with $|t|<n, A_{t}$ is specified. Put

$$
M_{t}=\sup \left\{|||f| \|: f \in A_{t}\right\}, \quad m_{t}=\inf \left\{\|\| f\|\|: f \in A_{t}\right\} .
$$

Let $f_{t} \in A_{t}$ satisfy $\left\|\mid f_{t}\right\| \|>\left(3 M_{t}+m_{t}\right) / 4$ and take two disjoint infinite subsets $N_{t 0}^{\prime}$ and $N_{t 1}^{\prime}$ of $N_{t} \backslash \operatorname{supp}\left(f_{t}\right)$ with $\mathbf{1}_{N_{t i}^{\prime}} \in X$, put $N_{t i}=N_{t i}^{\prime} \backslash\left\{k_{t i}\right\}$, and define

$$
A_{t i}=\left\{f \in A_{t}: f(n)=f_{t}(n) n \notin N_{t i}\right\} \quad(i=0,1) .
$$

Thus, by induction on $|t|$, we can obtain a family $\left\{A_{t}\right\}_{t \in T}$ of subsets of $X$, a family $\left\{f_{t}\right\}$ of elements of $X$, a family $\left\{N_{t}\right\}$ of infinite subsets of $N$, and a family of integers $\left\{k_{t}\right\}$ with the following properties.

(a) $A_{t i}$ is of the form

$$
A_{t i}=\left\{f \in A_{t}: f(n)=f_{t}(n), n \notin N_{t i}\right\} \quad(i=0,1),
$$

for each $t \in T$.

(b) $k_{t i} \in N_{t} \backslash N_{t i}$ and $f_{t}\left(k_{t}\right)=0$ for $t \in T$ and $i=0,1$.

(c) ||$f_{t} \| \mid>\left(3 M_{t}+m_{t}\right) / 4$, where $M_{t}$ and $m_{t}$ denote the supremum and infimum of $\left\{\|\mid f\| \|: f \in A_{t}\right\}$, respectively.

(d) $N_{s} \subset N_{t}$ whenever $t \prec s$ and $N_{t} \cap N_{s}=\varnothing$, if $s$ and $t$ are not comparable.

(e) $\operatorname{supp}\left(f_{t}-f_{s}\right) \subset N_{t} \backslash N_{s}$ for $t \prec s$.

By (e), $\left\{g_{t}\right\}_{t \in T}$, defined by

$$
g_{()}=f_{()}, \quad g_{t i}=f_{t i}-f_{t} \quad(i=0,1),
$$

is a disjoint family of elements of $X$. By the tree-completeness of $X$, there exists some $b \in\{0,1\}^{N}$ such that

$$
\left.f_{b}(x)=f_{(}\right)+\sum_{n \in N} g_{b \mid n}
$$

is in $X$. Let $\left\{k_{\alpha(n)}\right\}$ be a subsequence of $\left\{k_{b \mid n}\right\}$ such that $\mathbf{1}_{E} \in X$, where $E=\left\{k_{\alpha(1)}\right.$, $\left.k_{\alpha(2)}, \ldots\right\}$. Let $E_{n}=\left\{k_{\alpha(n)}, k_{\alpha(n+1)}, \ldots\right\}$ and $h_{n}=\mathbf{1}_{E_{n}}$. By (a) and (b), $g_{n+1}^{+}=f_{b}+h_{n+1}$ and $g_{n+1}^{-}=f_{b}-h_{n+1}$ are in $A_{b \mid n}$. Next, select some $\mu \in X^{*}$, such that $\mu\left(h_{1}\right)=1$ and $\mu(g)=0$ for each $g \in c_{0}$. Clearly, for such an element $\mu$ and each $n \in N$, we have $\mu\left(h_{n}\right)=1$. By 
(a), $2 f_{b}-f \in A_{b \mid n}$, thus $\left\|\left|2 f_{b \mid n}-f \|\right| \mid \leq M_{b \mid n}\right.$ for each $f \in A_{b \mid n}$ and $n \in N$. It follows that

$$
\frac{\left(3 M_{b \mid n-1}+m_{b \mid n-1}\right)}{2} \leq\left\|\left|2 f_{b \mid n}\right|\right\| \leq M_{b \mid n}+|||f|||, \quad \forall f \in A_{b \mid n},
$$

and so

$$
\frac{\left(3 M_{b \mid n-1}+m_{b \mid n-1}\right)}{2} \leq M_{b \mid n}+m_{b \mid n} \leq M_{b \mid n-1}+m_{b \mid n-1}, \quad \forall n \in N .
$$

Therefore,

$$
\begin{aligned}
M_{b \mid n}-m_{b \mid n} & \leq M_{b \mid n}-\frac{\left(M_{b \mid n-1}+m_{b \mid n-1}\right)}{2} \\
& \leq M_{b \mid n-1}-\frac{\left(M_{b \mid n-1}+m_{b \mid n-1}\right)}{2} \\
& =\frac{\left(M_{b \mid n-1}-m_{b \mid n-1}\right)}{2} .
\end{aligned}
$$

The above relations show that

$$
|||| g_{n+1}^{ \pm}|||-||| f_{b}|| \mid \leq M_{b \mid n}-m_{b \mid n} \leq \frac{\left(M_{b \mid n-1}-m_{b \mid n-1}\right)}{2} \leq \frac{\left(M_{()}-m_{()}\right)}{2^{n}}
$$

That is $\lim \left\|\left|g_{n}^{+}\left\|\left|=\left\||| f_{b}\right\|\|=\lim \|\right| g_{n}^{-}\right\|\right|\right.$. Moreover, $f_{b}=\left(g_{n}^{+}+g_{n}^{-}\right) / 2$. But weak$\lim \left(g_{n}^{+}-g_{n}^{-}\right) \neq 0$, since $\mu\left(h_{n}\right)=1$ for each $n \in N$. This shows that $X$ does not admit any wMLUR norm.

It is known that weakly midpoint locally uniformly rotundity of a Banach space $X$ is equivalent to saying that every point of $S(\hat{X})$ is an extreme point of $B\left(X^{* *}\right)$ [11]. It follows that the space considered in Theorem 2.1 has no equivalent norm such that $S(\hat{X})$ is a subset of $B\left(X^{* *}\right)$.

A norm on a Banach space $X$ is said to be strictly convex (rotund) (R) if the unit sphere of $X$ contains no nontrivial line segment. We say that a norm is Kadec if the weak and norm topologies coincide on the unit sphere. Every MLUR Banach space admits Kadec renorming (see [1]). Haydon in [6, Corollary 6.6] gives an example of a Kadec renormable space which has no equivalent $\mathrm{R}$ norm. The following result gives an example of a strictly convexifiable space with no equivalent Kadec norm.

COROLlary 2.2. If a tree-complete subspace $X$ of $\ell_{\infty}$ contains $c_{0}$, then it does not admit any equivalent Kadec renorming.

Proof. It is known that $\ell_{\infty}$ admits an equivalent strictly convex norm (see [4, page 120] or [2]). In [9] it is shown that every $\mathrm{R}$ Banach space with the Kadec property admits an equivalent MLUR renorming (see also [3, chapter IV]). Thus the result follows from Theorem 2.1. 


\section{REFERENCES}

[1] G. A. Aleksandrov and I. P. Dimitrov, On the equivalent weakly midpoint locally uniformly rotund renorming of the space $l_{\infty}$, Mathematics and Mathematical Education (Sunny Beach (Sl"nchev Bryag), 1985), B"lgar. Akad. Nauk, Sofia, 1985, pp. 189-191.

[2] M. M. Day, Normed Linear Spaces, 3rd ed., Springer-Verlag, New York, 1973.

[3] R. Deville, G. Godefroy, and V. Zizler, Smoothness and Renormings in Banach Spaces, Pitman Monographs and Surveys in Pure and Applied Mathematics, vol. 64, Longman Scientific \& Technical, Harlow, 1993.

[4] J. Diestel, Geometry of Banach Spaces-Selected Topics, Lecture Notes in Mathematics, vol. 485, Springer-Verlag, Berlin, 1975.

[5] R. Haydon, A nonreflexive Grothendieck space that does not contain $l_{\infty}$, Israel J. Math. 40 (1981), no. 1, 65-73.

[6] _ Trees in renorming theory, Proc. London Math. Soc. (3) 78 (1999), no. 3, 541-584.

[7] R. Haydon and V. Zizler, A new space with no locally uniformly rotund renorming, Canad. Math. Bull. 32 (1989), no. 1, 122-128.

[8] Z. Hu, W. B. Moors, and M. A. Smith, On a Banach space without a weak mid-point locally uniformly rotund norm, Bull. Austral. Math. Soc. 56 (1997), no. 2, 193-196.

[9] B.-L. Lin, P.-K. Lin, and S. L. Troyanski, Characterizations of denting points, Proc. Amer. Math. Soc. 102 (1988), no. 3, 526-528.

[10] J. Lindenstrauss, Weakly compact sets-their topological properties and the Banach spaces they generate, Symposium on Infinite-Dimensional Topology (Louisiana State Univ., Baton Rouge, La, 1967), Ann. of Math. Studies, No. 69, Princeton University Press, Princeton, NJ, 1972, pp. 235-273.

[11] W. B. Moors and J. R. Giles, Generic continuity of minimal set-valued mappings, J. Austral. Math. Soc. Ser. A 63 (1997), no. 2, 238-262.

A. K. Mirmostafaee: Department of Mathematics, Damghan University of Sciences, P.O. Box 36715-364, Damghan, Iran 


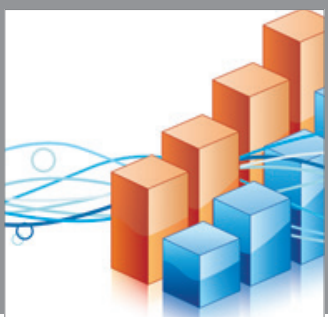

Advances in

Operations Research

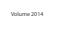

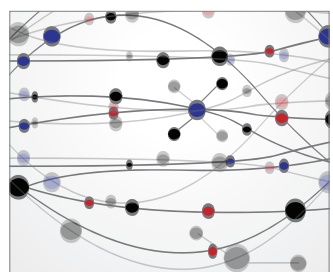

\section{The Scientific} World Journal
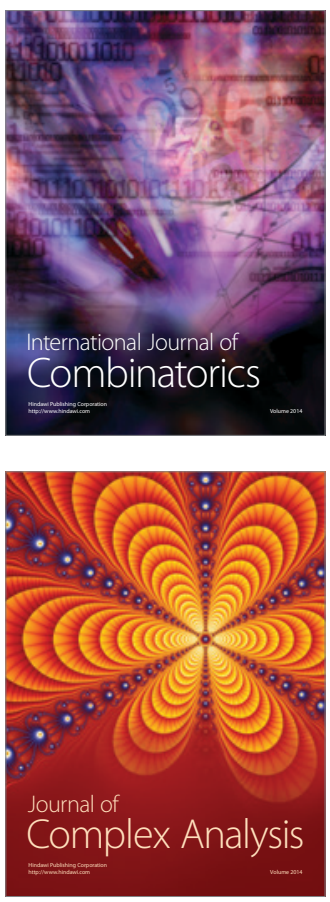

International Journal of

Mathematics and

Mathematical

Sciences
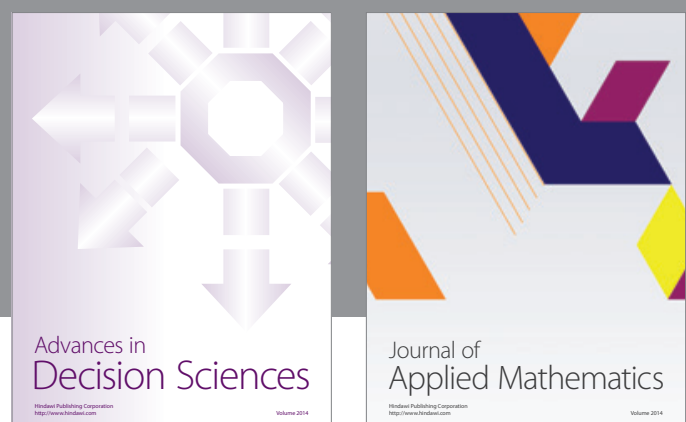

Journal of

Applied Mathematics
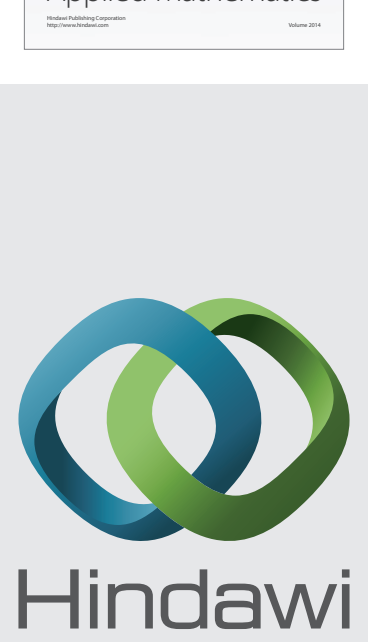

Submit your manuscripts at http://www.hindawi.com
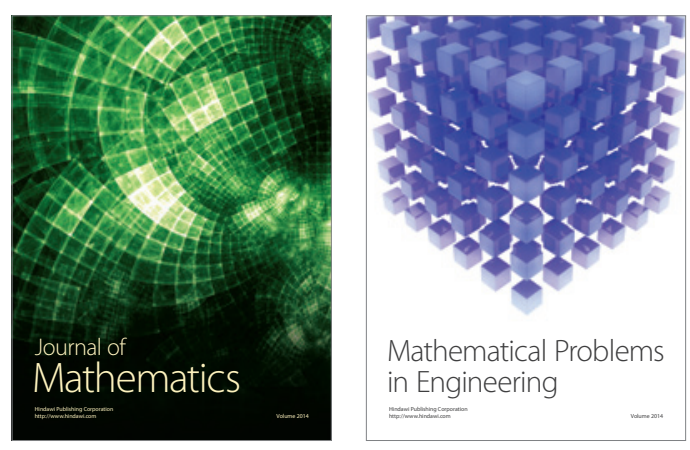

Mathematical Problems in Engineering
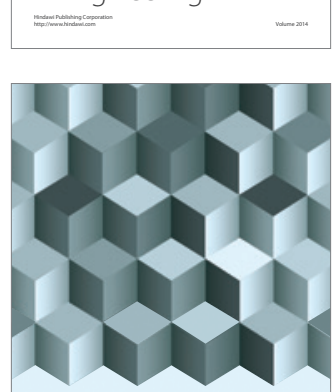

Journal of

Function Spaces
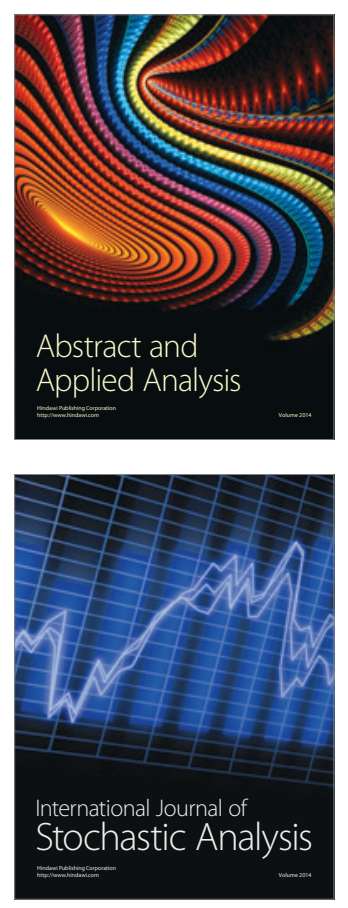

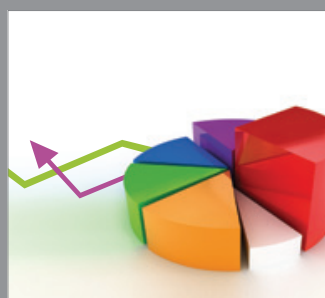

ournal of

Probability and Statistics

Promensencen
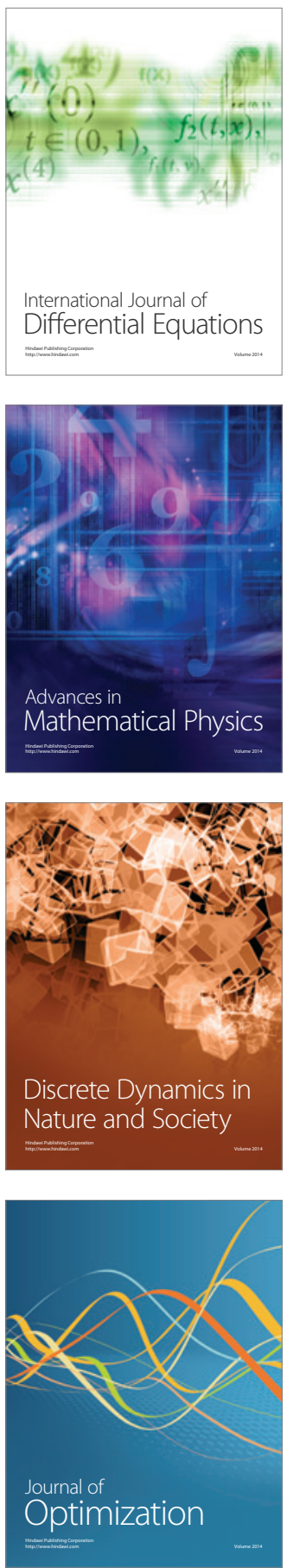